\title{
Multi-component generalized mode-coupling theory: predicting dynamics from structure in glassy mixtures
}

\author{
Simone Ciarella ${ }^{1,2, a}{ }_{\mathbb{D}}$, Chengjie Luo $^{1}$, Vincent E. Debets ${ }^{1}$, and Liesbeth M. C. Janssen ${ }^{1, \mathrm{~b}}{ }_{\mathbb{C}}$ \\ ${ }^{1}$ Soft Matter and Biological Physics, Department of Applied Physics, Eindhoven University of Technology, P.O. Box 513, \\ 5600, MB, Eindhoven, The Netherlands \\ 2 Laboratoire de Physique de l'Ecole Normale Supérieure, ENS, Université PSL, CNRS, Sorbonne Université, Université de \\ Paris, 75005 Paris, France
}

Received 31 March 2021 / Accepted 21 June 2021 / Published online 6 July 2021

(C) The Author(s) 2021

\begin{abstract}
The emergence of glassy dynamics and the glass transition in dense disordered systems is still not fully understood theoretically. Mode-coupling theory (MCT) has shown to be effective in describing some of the non-trivial features of glass formation, but it cannot explain the full glassy phenomenology due to the strong approximations on which it is based. Generalized mode-coupling theory (GMCT) is a hierarchical extension of the theory, which is able to outclass MCT by carefully describing the dynamics of higher-order correlations in its generalized framework. Unfortunately, the theory has so far only been developed for single-component systems and as a result works poorly for highly polydisperse materials. In this paper, we solve this problem by developing GMCT for multi-component systems. We use it to predict the glassy dynamics of the binary Kob-Andersen Lennard-Jones mixture, as well as its purely repulsive Weeks-Chandler-Andersen analogue. Our results show that each additional level of the GMCT hierarchy gradually improves the predictive power of GMCT beyond its previous limit. This implies that our theory is able to harvest more information from the static correlations, thus being able to better understand the role of attraction in supercooled liquids from a first-principles perspective.
\end{abstract}

\section{Introduction}

Understanding how supercooled liquids become rigid and turn into amorphous solids is still one of the major challenges in condensed matter physics [1-4]. This socalled glass transition is not a transition in the thermodynamic sense [5], but it is defined by the dramatic increase in viscosity (or relaxation time) upon only a relatively slight change in thermodynamic control parameters, e.g., temperature or density $[6,7]$. This sudden and highly nonlinear dynamic response is accompanied by only subtle changes in the microscopic structure, rendering it difficult to identify the main physical mechanisms underlying the glass transition [8-12].

In principle, it is widely accepted that the dynamics of each material is ultimately related to its structure [13], and numerous theories have also aimed to exploit this idea to describe the glass transition [14-22]. Among these, mode-coupling theory (MCT) stands out as one of the few theories which is entirely based on first principles $[8,19,20,23]$. This theory seeks to predict the

Simone Ciarella, Chengjie Luo, and Vincent E. Debets contributed equally to this study.

\footnotetext{
${ }^{\mathrm{a}}$ e-mail: simoneciarella@gmail.com (corresponding author)

b e-mail: l.m.c.janssen@tue.nl
}

full microscopic relaxation dynamics of a glass-forming system (as a function of time, temperature, density, and wavenumber $k$ ) based solely on knowledge of simple structural material properties, such as the static structure factor $S(k)$. Although the theory is often not fully quantitatively accurate, MCT has enjoyed success in predicting, e.g., multi-step relaxation patterns and universal scaling laws in the dynamics, stretched exponentials, and growing dynamical length scales [24-26]. Furthermore, the theory offers a qualitative and physically intuitive account of glass formation in terms of the so-called cage effect [25]. Further extensions of MCT such as the stochastic beta relaxation theory can even explain the crossover from a power law to exponential growth of the relaxation time $[21,22]$. The (most severe) limitation of MCT lies, however, in its assumption of Gaussian correlations which causes noticeable discrepancy between the theory and experiments.

So far, promising methodical MCT correction efforts have been put forward for single-component systemsor equivalently systems with a small degree of polydispersity - using higher-order field-theoretic loop expansions [27-32]. Results show that such an expansion can indeed be accomplished, producing a novel, hierarchical first-principles theory known as generalized MCT (GMCT). By systematically developing the hierarchical equations, GMCT has already proven to be capable of predicting the microscopic dynamics of glassy mate- 
rials with near-quantitative accuracy in the low to moderately supercooled regime [29]. Similar to MCT, this generalized framework also requires only static structure as input and has no free parameters. Furthermore, GMCT also provides predictive insights into regimes of previously inaccessible dynamics for single-component glassy systems $[29,33,34]$, and notably preserves the celebrated scaling laws of standard MCT [33,34].

Unfortunately, single-component systems are a poor representation of most studied glasses, which are typically polydisperse and as a result exhibit different overall dynamics compared to monodisperse systems $[35,36]$. In experiments, polydispersity is for the most part inevitable, while in computer simulations it is often added to hinder and prevent crystal formation [37]. Noticeably, techniques such as Monte Carlo (MC) swaps capitalize onto polydispersity to achieve faster relaxation dynamics and explore the free energy landscape in uncommon ways [38]. Binary polydisperse systems are the simplest generalization of singlecomponent systems in this direction. They add only a supplementary component to the mix and are able to retain most of the advantages of a polydisperse system while adding the least possible complexity.

In this paper, we set out to extend the GMCT framework to systems with an arbitrary number of species, and seek to apply the newly developed framework to describe arguably the most famous and simple examples of binary glassy systems: the Kob-Andersen binary Lennard-Jones (KABLJ) mixture [37] and its purely repulsive version based on the Weeks-ChandlerAndersen (WCA) potential [39]. These systems have been extensively studied in the past and comparisons with MCT have identified the existence of a region where MCT is already in its non-ergodic phase, thus predicting a glass, while simulations at the same temperature and density indicate a supercooled liquid phase $[16,40-44]$. In other words, a discrepancy still exists between the simulations and MCT, even when MCT is extended to multi-component systems [45-49]. Here, after demonstrating the ability of GMCT to systematically tackle this discrepancy, we will also address a fundamental question regarding the simplest ingredients required to describe the dynamics of binary supercooled liquids. Due to the fact that standard MCTwhich is based solely on $S(k)$-fails in predicting the precise location of the glass transition, it could be concluded that higher-order correlations are required $[40,41,44]$. However, GMCT can circumvent this failure. It again uses only $S(k)$ as input, but in a more refined set of equations which can translate structural properties into dynamical ones in a more accurate manner. Applying our multi-component GMCT to both mixtures, we will conclude that each level of the GMCT hierarchy provides a significant improvement in the prediction of the glass transition, finally conjecturing that the infinite hierarchy might be able to accurately predict the glassy dynamics from $S(k)$ only.

\section{Multi-component GMCT}

Multi-component GMCT is derived starting from the Mori-Zwanzig approach $[50,51]$ to predict the dynamics of density correlations, similarly to standard MCT. However, while standard MCT amounts to a single integro-differential equation closed by a factorization approximation $[8,19,20,23,47]$, GMCT is a hierarchy of nested integro-differential equations [29]. Each level of this hierarchy represents an MCT-like dynamical equation for a higher-order, multi-point density correlation function, which we recursively solve and use to predict the dynamics of the correlations at the lower levels. Since a solution of this hierarchy is well defined for any self-consistent closure or truncation of the hierarchy [52], we can formally continue the GMCT scheme up to arbitrary order to include as many higher-order correlations as desired.

In the Supplementary Information, we report the full derivation of multi-component GMCT for an $M$ component mixture. To summarize it here, we introduce the main objects of the theory, i.e., the speciesdependent density modes:

$$
\rho_{\mathbf{q}}^{\alpha}(t)=\frac{1}{\sqrt{N}} \sum_{i=1}^{N_{\alpha}} e^{-i \mathbf{q} \cdot \mathbf{r}_{i}^{\alpha}(t)} .
$$

Here, $\mathbf{q}$ is a wavevector of length $q=|\mathbf{q}|, t$ is the time, the index $\alpha$ represents one of the $M$ species, $N_{\alpha}$ is the number of particles that belong to the species $\alpha$, and $N=\sum_{\alpha=1}^{M} N_{\alpha}$ is the total number of particles in the system. To simplify our equations we introduce the notation that $\left\{x_{i}\right\}$ is a list $x_{1}, \ldots, x_{n}$ and $\left\{x_{i}\right\} / x_{j}$ is the same ordered list $\left\{x_{i}\right\}$ where the specific element $x_{j}$ has been removed. In solving multi-component GMCT we are interested in determining the dynamical equation of the density correlations of order $n$. These dynamical correlations are defined as

$$
F_{\left\{\alpha_{i}\right\} ;\left\{\beta_{i}\right\}}^{(n)}\left(\left\{k_{i}\right\}, t\right)=\left\langle\rho_{-\mathbf{k}_{1}}^{\alpha_{1}} \cdots \rho_{-\mathbf{k}_{n}}^{\alpha_{n}} \rho_{\mathbf{k}_{1}}^{\beta_{1}}(t) \cdots \rho_{\mathbf{k}_{n}}^{\beta_{n}}(t)\right\rangle,
$$

where the angular brackets denote an ensemble average. In particular, when the order $n=1$, the correlation corresponds to the intermediate scattering function. The only required input of the theory (aside from temperature and density) is the set of wavevector-dependent static correlations $S^{(n)}$,

$$
\begin{aligned}
S_{\left\{\alpha_{i}\right\} ;\left\{\beta_{i}\right\}}^{(n)}\left(\left\{k_{i}\right\}\right) & =F_{\left\{\alpha_{i}\right\} ;\left\{\beta_{i}\right\}}^{(n)}\left(\left\{k_{i}\right\}, t=0\right) \\
& \approx \prod_{i=1}^{n} S^{\alpha_{i} \beta_{i}}\left(k_{i}\right),
\end{aligned}
$$

which defines the full microstructure of the system at any given temperature and density. Here, we factorize these static correlations as products of the two-point correlation $S^{\alpha \beta}(k) \equiv S_{\alpha ; \beta}^{(1)}(k)$, which is also known as 
the static structure factor. This means that all the predictions of the theory are based on the structure factor only. As such, we will conclude later that all the predicted differences in dynamics among the two supercooled liquid systems are already encoded in their static structure factors.

Within our multi-component GMCT hierarchy, each dynamical correlation function $F_{\left\{\alpha_{i}\right\} ;\left\{\beta_{i}\right\}}^{(n)}\left(\left\{k_{i}\right\}, t\right)$ obeys the following equation of motion:

$$
\begin{aligned}
& \ddot{F}_{\left\{\alpha_{i}\right\} ;\left\{\beta_{i}\right\}}^{(n)}\left(\left\{k_{i}\right\}, t\right)+\sum_{\left\{\gamma_{i}\right\}} \mu_{\left\{\alpha_{i}\right\} ;\left\{\gamma_{i}\right\}} \dot{F}_{\left\{\gamma_{i}\right\} ;\left\{\beta_{i}\right\}}^{(n)}\left(\left\{k_{i}\right\}, t\right) \\
& \quad+\sum_{\left\{\gamma_{i}\right\}\left\{\theta_{i}\right\}} F_{\left\{\alpha_{i}\right\} ;\left\{\gamma_{i}\right\}}^{(n)}\left(\left\{k_{i}\right\}, t\right)\left(S^{(n)}\right)_{\left\{\gamma_{i}\right\} ;\left\{\theta_{i}\right\}}^{-1} \\
& \quad\left(\left\{k_{i}\right\}\right) J_{\left\{\theta_{i}\right\} ;\left\{\beta_{i}\right\}}^{(n)}\left(\left\{k_{i}\right\}\right) \\
& \quad+\int_{0}^{t} d \tau \sum_{\left\{\gamma_{i}\right\}\left\{\theta_{i}\right\}} \dot{F}_{\left\{\alpha_{i}\right\} ;\left\{\gamma_{i}\right\}}^{(n)}\left(\left\{k_{i}\right\}, t-\tau\right)\left(J^{(n)}\right)_{\left\{\gamma_{i}\right\} ;\left\{\theta_{i}\right\}}^{-1}\left(\left\{k_{i}\right\}\right) \\
& K_{\left\{\theta_{i}\right\} ;\left\{\beta_{i}\right\}}^{(n)}\left(\left\{k_{i}\right\}, \tau\right)=0 .
\end{aligned}
$$

where the effective friction coefficient matrix $\mu_{\left\{\alpha_{i}\right\} ;\left\{\beta_{i}\right\}}$ is assumed to be diagonal and species independent, i.e., $\mu_{\left\{\alpha_{i}\right\} ;\left\{\beta_{i}\right\}}=\mu \prod_{i} \delta_{\alpha_{i} \beta_{i}}$. The matrices $J$ are static elements defined as

$$
\begin{gathered}
J_{\left\{\alpha_{i}\right\} ;\left\{\beta_{i}\right\}}^{(n)}\left(\left\{k_{i}\right\}\right)=\left\langle\frac{d}{d t}\left[\rho_{k_{1}}^{\alpha_{1}} \cdots \rho_{k_{n}}^{\alpha_{n}}\right] \mid \frac{d}{d t}\left[\rho_{k_{1}}^{\beta_{1}} \cdots \rho_{k_{n}}^{\beta_{n}}\right]\right\rangle \\
\approx \sum_{i=1}^{n} \delta_{\alpha_{i}, \beta_{i}} \frac{k_{B} T x_{\alpha_{i}} k_{i}^{2}}{m_{\alpha_{i}} S^{\alpha_{i} \beta_{i}}\left(k_{i}\right)} \prod_{j=1}^{n} S^{\alpha_{j} \beta_{j}}\left(k_{j}\right)
\end{gathered}
$$

with $T$ the temperature, $m_{\alpha}$ the particle mass of type $\alpha$, and $x_{\alpha}=N_{\alpha} / N$ their number ratio. Each level $n$ of the hierarchy is connected to the next via the memory term

$$
\begin{aligned}
& K_{\left\{\alpha_{i}\right\} ;\left\{\beta_{i}\right\}}^{(n)}\left(\left\{k_{i}\right\}, \tau\right)=\frac{\rho}{2} \sum_{\mu^{\prime} \nu^{\prime}} \sum_{\mu \nu} \\
& \quad \int \frac{d \mathbf{q}}{(2 \pi)^{3}} \cdot \sum_{j=1}^{n} \frac{k_{B} T}{m_{\alpha_{j}}} \mathcal{V}_{\mu^{\prime} \nu^{\prime} \alpha_{j}}\left(\mathbf{q}, \mathbf{k}_{\mathbf{j}}-\mathbf{q}, \mathbf{k}_{\mathbf{j}}\right) \\
& F_{\mu^{\prime}, \nu^{\prime},\left\{\alpha_{i}\right\} / \alpha_{j} ; \mu, \nu,\left\{\beta_{i}\right\} / \beta_{j}}^{(n+1)}\left(\mathbf{q}, \mathbf{k}_{\mathbf{j}}-\mathbf{q},\left\{k_{i}\right\} / k_{j}, \tau\right) \\
& \cdot \mathcal{V}_{\mu \nu \beta_{j}}\left(\mathbf{q}, \mathbf{k}_{\mathbf{j}}-\mathbf{q}, \mathbf{k}_{\mathbf{j}}\right) \frac{k_{B} T}{m_{\beta_{j}}} .
\end{aligned}
$$

Here, $\mathcal{V}_{\alpha \beta \gamma}(\mathbf{q}, \mathbf{k}-\mathbf{q}, \mathbf{k})$ is the static vertex function, which remains equal to the standard one of MCT [47] and physically represents the coupling strength among different wavevectors. Explicitly, the vertex function reads

$$
\begin{gathered}
\mathcal{V}_{\alpha \beta \gamma}(\mathbf{q}, \mathbf{k}-\mathbf{q}, \mathbf{k})=\delta_{\beta \gamma} \mathbf{q} \cdot \mathbf{k} c_{\alpha \gamma}(q) \\
+\delta_{\alpha \gamma}(\mathbf{k}-\mathbf{q}) \cdot \mathbf{k} c_{\beta \gamma}(|\mathbf{k}-\mathbf{q}|)
\end{gathered}
$$

with the direct correlation function $c_{\alpha \beta}(q)$. It relates to the static structure factors via the Ornstein-Zernike equation $c_{\alpha \beta}(q)=\rho^{-1}\left(\delta_{\alpha \beta} / x_{\alpha}-\left(\mathbf{S}^{-1}(q)\right)_{\alpha \beta}\right.$ where $\rho=$ $N / V$ is the number density of the system [53]. Finally, we note that in this derivation we have assumed the convolution approximation for static three-point correlators [54,55] and neglected the so-called off-diagonal correlations, similar to what is done in conventional MCT and single-component GMCT [8, 19, 20, 56]. Equation 4 is subject to the initial boundary conditions $\dot{F}_{\left\{\alpha_{i}\right\} ;\left\{\beta_{i}\right\}}^{(n)}\left(\left\{k_{i}\right\}, t=0\right)=0$ and $F_{\left\{\alpha_{i}\right\} ;\left\{\beta_{i}\right\}}^{(n)}\left(\left\{k_{i}\right\}, t=0\right)=$ $S_{\left\{\alpha_{i}\right\} ;\left\{\beta_{i}\right\}}^{(n)}\left(\left\{k_{i}\right\}\right)$ (Eq. 3) for all $\left\{\alpha_{i}\right\},\left\{\beta_{i}\right\}$, and $\left\{k_{i}\right\}$.

In principle, the above hierarchical equations can be solved up to arbitrary order $n$, but in practice we must apply a suitable closure at finite order $n_{\max }$ to obtain numerically tractable results. We use the following mean-field closure at level $n_{\max }>2[29]$

$$
\begin{aligned}
& K_{\left\{\alpha_{i}\right\} ;\left\{\beta_{i}\right\}}^{\left(n_{\max }-1\right)}\left(\left\{k_{i}\right\}, t\right) \approx \frac{1}{n_{\max }-2} \\
& \sum_{j} K_{\left\{\alpha_{i}\right\} / \alpha_{j} ;\left\{\beta_{i}\right\} / \beta_{j}}^{\left(n_{\max }-2\right)}\left(\left\{k_{i}\right\} / k_{j}, t\right) F_{\alpha_{j} ; \beta_{j}}^{(1)}\left(k_{j}, t\right) .
\end{aligned}
$$

Note that this makes use of the permutation invariance of all wavenumber arguments $\left\{k_{1}, \ldots, k_{n}\right\}$. In terms of the intermediate scattering function the closure is equivalent to the factorization approximation $F^{\left(n_{\max }\right)}(t) \sim F^{\left(n_{\max }-1\right)}(t) \times F^{(1)}(t)$. Hence, at $n_{\max }=$ 2 we obtain the same closure as in standard multicomponent MCT [47]. As a reminder, most of the problems with standard MCT come from the fact that such a factorization closure is too strong and unjustified [19,20,23]. Multi-component GMCT allows us instead to shift the closure to a larger $n_{\max }>2$, meaning that the correlations $F^{\left(n^{\prime}\right)}(t)$ of order $n^{\prime}<n_{\max }$ are not factorized and are more correctly described. This approach has already been shown to be beneficial in single-component glassy systems [27-29,33,34], and in this paper, we demonstrate that a similar improvement can be gained for binary systems.

\section{Numerical details}

\subsection{Numerical solution of GMCT}

Since the system is isotropic and invariant under rotations, we use spherical coordinates to transform the three-dimensional integrals over $\mathbf{q}$ that appear in any memory function of the hierarchy (Eq. 6) as a double integral over $q=|\mathbf{q}|$ and $p=|\mathbf{k}-\mathbf{q}|$. Then, $q$ is discretized over a uniformly spaced grid of $N_{k}=100$ points $q=q_{0}+\hat{q} \Delta q$ with $\hat{q}=0,1, \ldots, N_{k}-1$ and $\Delta q=40 / N_{k}$. In the Supplementary Information, we show that a grid of $N_{k}>70$ wavenumbers is sufficiently converged to predict the MCT critical point. This choice of parameters allows us to replace the double integral by Riemann sums 


$$
\int_{0}^{\infty} d k \int_{|q-k|}^{q+k} d p \rightarrow(\Delta q)^{2} \sum_{\hat{k}=0}^{N_{k}-1} \sum_{\hat{p}=|\hat{q}-\hat{k}|}^{\min \left[N_{k}-1, \hat{q}+\hat{k}\right]}
$$

Following Ref. [57], we set $q_{0}=\Delta q / 2$ in order to prevent any possible divergence for $q \rightarrow 0$.

To obtain time-dependent solutions for our GMCT equations, we set the effective friction coefficient $\mu=1$, assume overdamped conditions by dropping the secondorder derivative $\ddot{F}^{(n)}$ in Eq. 4 (since it only affects the short-time dynamics), and start with a Taylor expansion around $t=0$ for all dynamical correlation functions $F^{(n)}$ up to the level $n_{\max }-1$; the correlator at the highest level, $F^{\left(n_{\max }\right)}$, follows from our closure relation, Eq. 8. We then integrate Eq. 4 in time using Fuchs' algorithm [58], where the first $N_{t}=64$ time points are calculated with a step size of $\Delta t=10^{-6}$, and $\Delta t$ is subsequently doubled every $N_{t} / 2$ points. At each point in time, we iteratively update the wavevector-dependent memory kernels (Eq. 6) for all $n \leq n_{\max }$ until convergence. Note that in these GMCT equations, the partial static structure factors $S^{\alpha \beta}(k)$ enter both in the initial boundary conditions for $F^{(n)}$, as well as in the static vertices and the matrices $J^{(n)}$. In summary, at any given $(T, \rho)$ we only require $S^{\alpha \beta}(k)$ as input to predict the microscopic relaxation dynamics of the system. While our GMCT framework gives access to all multipoint dynamical density correlations up to order $n_{\max }$, in the following we shall restrict the discussion to the intermediate scattering function $F^{\alpha \beta}(k, t)=F_{\alpha ; \beta}^{(1)}(k, t)$.

\subsection{Numerical simulations}

We use multi-component GMCT to predict the glassy behavior of two binary mixtures: the Kob-Andersen binary Lennard-Jones (LJ) mixture [37] and its WeeksChandler-Anderson truncation (WCA) [39]. Both are three-dimensional 80:20 mixtures of particles $A: B$ which interact with each other via the following potential

$$
V_{\alpha \beta}(r)= \begin{cases}4 \epsilon_{\alpha \beta}\left[\left(\frac{\sigma_{\alpha \beta}}{r}\right)^{12}-\left(\frac{\sigma_{\alpha \beta}}{r}\right)^{6}+C_{\alpha \beta}\right], & r \leq r_{\alpha \beta}^{c}, \\ 0, & r>r_{\alpha \beta}^{c} .\end{cases}
$$

Here, the cutoff radius $r_{\alpha \beta}^{c}$ is $2.5 \sigma_{\alpha \beta}$ for LJ, while it corresponds to the potential minimum for WCA [39]. The constant $C_{\alpha \beta}$ ensures that $V_{\alpha \beta}\left(r_{\alpha \beta}^{c}\right)=0$. We use $\epsilon_{A A}=1, \epsilon_{A B}=1.5, \epsilon_{B B}=0.5, \sigma_{A A}=1, \sigma_{A B}=$ $0.8, \sigma_{B B}=0.88$ to obtain good glass-forming mixtures [37].

In order to calculate the relevant quantities we need for a comparison with multi-component GMCT, we perform molecular dynamics simulations in the NVE ensemble using HOOMD-blue [59]. We properly equilibrate both systems at different densities $\rho$ and temperatures $T$. Periodic boundary conditions are imposed and the box size is set at a length $L=10$ so that the density is tuned via the number of particles $N=1200,1400$ and 1600. All parameters and results are reported in terms of reduced WCA units [39]. From the simulation trajectories, we calculate the partial static structure factors $S^{\alpha \beta}(k)$ and the collective intermediate scattering functions $F^{\alpha \beta}(k, t)$. For the multi-component GMCT calculations, we use the simulated $S^{\alpha \beta}(k)$ as the input of Eq. 4 to predict the theoretical $F^{\alpha \beta}(k, t)$. In the next section, we compare the output of multi-component GMCT with the $F^{\alpha \beta}(k, t)$ obtained from simulation, and show that multi-component GMCT becomes progressively closer to the simulated glass transition temperature as we increase the level of the GMCT hierarchy.

\section{Results and discussion}

\subsection{From structure to dynamics}

The strength of GMCT is its capability of predicting dynamics from statics. The first result that we show underlines the sensitivity of GMCT to small variations in the static structure. In Fig. 1a, we compare the partial structure factors (normalized for visual purposes) of the binary LJ (yellow) and binary WCA (gray) systems at density $\rho=1.2$ and temperature $T=0.65$, which corresponds to low density in the supercooled regime. Notice that all the components of the structure factor are very similar between the two mixtures, consistent with previous simulations [40-42,44]. However, as shown in Fig. 1c, and also in agreement with earlier studies $[40-42,44]$, the simulated relaxation dynamics of the two systems (blue curves) differ significantly. In particular, the structural relaxation of $F^{A A}\left(k_{\text {peak }}, t\right)$, with $k=k_{\text {peak }}$ corresponding to the main peak of $S^{A A}(k)$, is approximately one order of magnitude slower for the LJ mixture. This disparity in dynamics also becomes more pronounced when decreasing $T$.

Importantly, binary MCT can only partly account for these dynamical differences based on the input static structure factors, and furthermore, the standard theory cannot reach quantitative accuracy for either system at any given temperature 3 [45-48]. Indeed, it is also demonstrated in Fig. 1c that binary MCT (red curves) fails to predict the correct dynamics at this temperature and density, erroneously predicting a non-ergodic glass phase for both systems. Our multi-component GMCT framework, on the other hand, better approaches the simulated long-time dynamics from the same $S^{\alpha \beta}(k)$ as input as we increase the level of the hierarchy $n_{\max }$. In particular, note that the highest considered GMCT closure level, $n_{\max }=4$ (green curves), correctly yields an ergodic phase for both systems, with the LJ mixture having one to two orders of magnitude slower relaxation dynamics than the WCA mixture. This prediction is in good qualitative agreement with simulation.

When the density is high $(\rho=1.6)$ the attraction that distinguishes LJ from WCA is less significant, since all particles predominantly probe only the short-range 

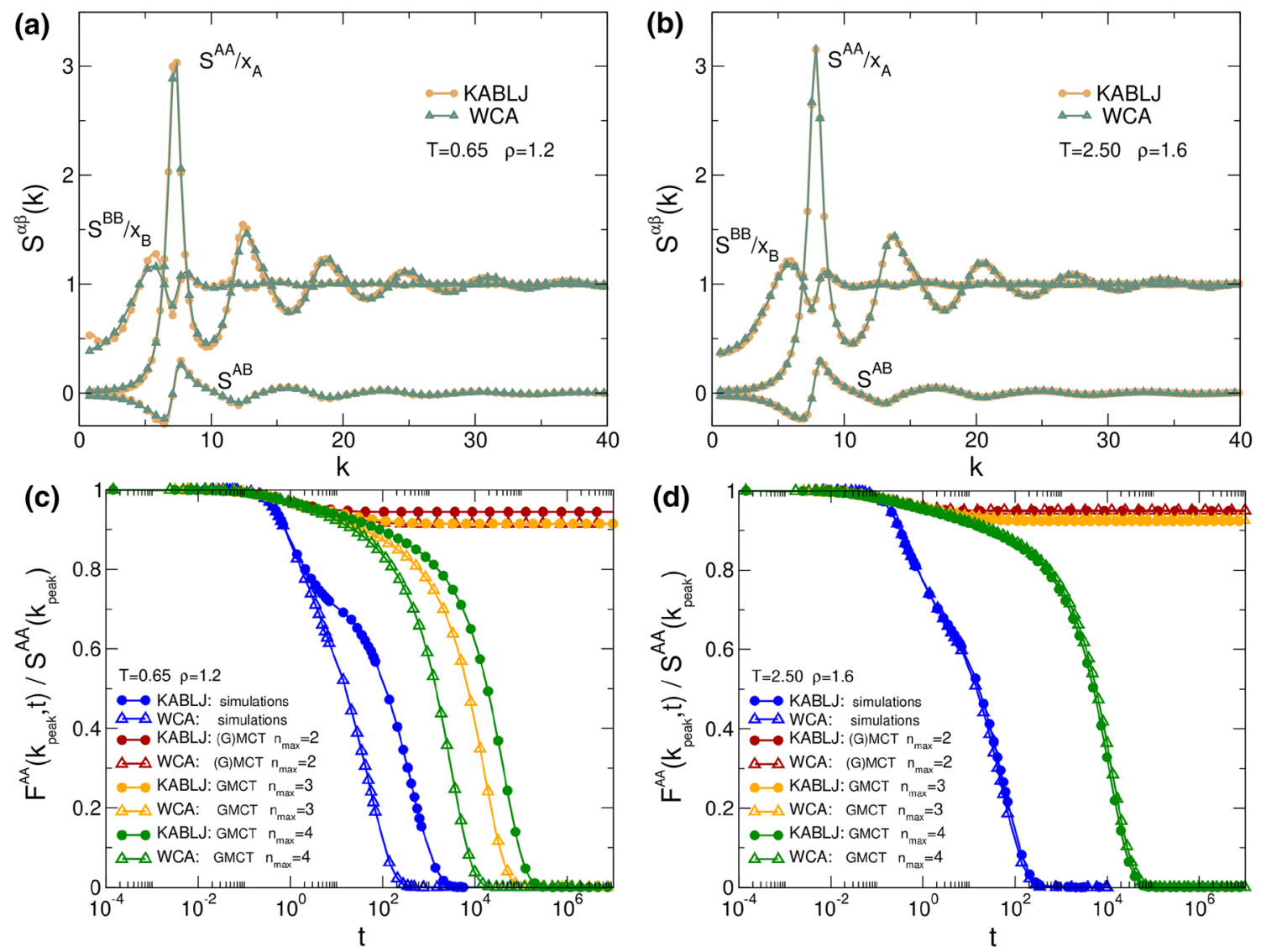

Fig. 1 Structure and dynamics of supercooled binary LJ and WCA mixtures at $T=0.65, \rho=1.2$ and $T=2.50, \rho=$ 1.6. In panels $\mathbf{a}$ and $\mathbf{b}$, we compare the partial static structure factors (where the $A A$ and $B B$ components are normalized for visual purposes) of binary LJ (yellow) and binary WCA (gray). In $\mathbf{c}$ and $\mathbf{d}$, we show the dynamics in the supercooled regime of the component $F^{A A}(k, t)$, which is the term that dominates the dynamics since $80 \%$ of the system

repulsive regime. In fact, we see in Fig. $1 b$ that all the components of $S^{\alpha \beta}(k)$ are virtually identical among the two mixtures. The dynamics in this supercooled regime, reported in Fig. 1d, is also almost the same for the simulated mixtures. Notice that the value of $T=2.5$ corresponds to approximately $1.5 T_{g}$, and it is comparable to the value of $T=0.65$ at $\rho=1.2$ of Fig. 1c. Similarly, every level of the binary GMCT hierarchy also predicts almost indistinguishable dynamics at this density. However, it is once again noticeable that a higher $n_{\max }$ makes multi-component GMCT converge toward the simulations.

Overall, Fig. 1 clearly shows that small differences in the structure are captured by multi-component GMCT and amplified to predict the dynamics in the glassy regime. While on the one hand this sensitivity of the theory means that a high precision is required when measuring the input-S(k), on the other hand this supports the idea that important information about the dynamics is already enclosed in static 2-point density correlations $[42,44]$.

is type $A$. The wavenumber $k=k_{\text {peak }}$ corresponds to the maximum of $S^{A A}(k)$. The different curves in panels (c) and (d) show $F^{A A}(k, t)$ measured from MD simulations (blue), binary MCT (red), binary GMCT with $n_{\max }=3$ (orange), and binary GMCT with $n_{\max }=4$ (green). When increasing the level of the GMCT hierarchy $n_{\max }$, the $F^{A A}(k, t)$ predicted by multi-component GMCT tends to converge to the simulation results

\subsection{The role of polydispersity}

By extending the framework of GMCT to include multiple components, we can directly account for polydispersity, i.e., the heterogeneity of sizes of molecules or particles in a mixture. It is ubiquitous in experiments at the colloidal scale because two particles are hardly equal and in the context of glasses it is also useful to avoid crystallization [9]. Furthermore, it has been shown that even in simulations where monodispersity is possible, it can be beneficial to use polydispersity in order to employ algorithms such as Monte Carlo swaps which can significantly improve the performance of computations [38].

If the degree of polydispersity is small it has been shown that single-component GMCT is capable of very accurate predictions [29]. However, for highly polydisperse systems or complex architectures [60-63] singlecomponent theories require a pre-averaging of the structure. This can severely influence their predictions. In particular, since $(\mathrm{G}) \mathrm{MCT}$ is very sensitive to the value 
of the main peak of the static structure factor [60], averaging the $A A$ correlation with the $A B$ and $B B$ components inevitably leads to a decrease in such peak which, in turn, alters the results of $(\mathrm{G}) \mathrm{MCT}$.

In Fig. 2, we examine the consequences of approximating a highly polydisperse system, i.e., our binary LJ and WCA mixtures, as being effectively monodisperse. We report the relaxation time $\tau$ as a function of the inverse temperature at $\rho=1.2$, comparing simulations to single-component and multi-component GMCT; for single-component GMCT we use the average static structure factors $S(k)$ as input, whereas for multicomponent GMCT we explicitly distinguish between all the partial components $S^{\alpha \beta}(k)$ (see Fig. S2 for a comparison between the pre-averaged monodisperse structure factor and the binary ones). The relaxation time $\tau$ is defined as

$$
F^{A A}\left(k_{\text {peak }}, t=\tau\right)=\frac{F^{A A}\left(k_{\text {peak }}, 0\right)}{e},
$$

which grows rapidly during supercooling toward the glass transition temperature $T_{g}[9]$. We find that singlecomponent (G)MCT significantly underestimates the critical glass transition temperature for both systems. In particular, at the supercooled temperatures where the simulated $\tau$ reaches a value of $\tau \sim 10^{4}-10^{5}$ (i.e., near the simulated $T_{g}$ ), we find that our singlecomponent (G)MCT approximation yields a relaxation time that is almost three orders of magnitude too low. This underestimation of the glassy dynamics is also consistent with MCT studies of polymeric systems that use pre-averaged static structure factors [60-63]. Moreover, note that the qualitative shape of the $\tau(T)$ curves predicted by single-component theory also deviates markedly from the simulation results, and that little improvement is gained by increasing $n_{\max }$.

By contrast, when properly taking into account the binary nature of both systems, multi-component (G)MCT yields predictions that more closely resemble the $\tau(T)$ simulation curves, at least on a qualitative level. We also see that the multi-component theory in fact overestimates the critical temperature, with the highest overestimation found for the lowest $n_{\max }$. This general tendency to overestimate the glassiness is also consistent with other multi-component $[47,48]$ and standard MCT [20] calculations. Overall, these results underline the fact that non-trivial couplings exist in the structure and dynamics of multi-component glassy mixtures, highlighting the need to explicitly account for polydispersity in such systems.

\subsection{Relaxation time}

We proceed by comparing our numerical simulations with the predictions of multi-component GMCT. The comparison is summarized in Fig. 3 where we report the relaxation time $\tau$ as a function of the inverse temperature for three different bulk densities. It should be noted that here we solely focus on $\alpha=\beta=A$ when determining $\tau$, because particles of type $A$ constitute $80 \%$ of

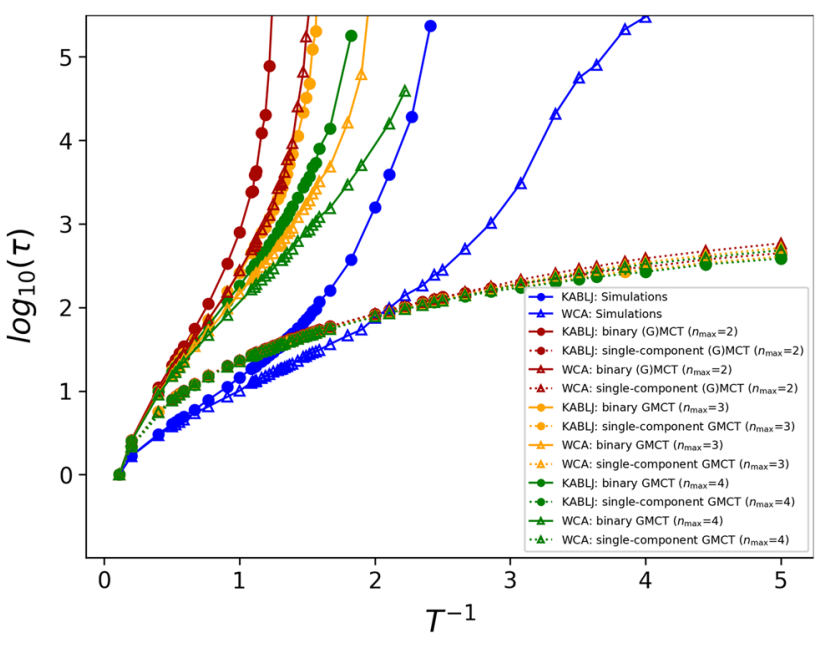

Fig. 2 Relaxation time as a function of inverse temperature from simulations (blue), single component (dashed lines), and multi-component (continuous line) (G)MCT, for binary LJ and WCA at $\rho=1.2$. The data show that singlecomponent GMCT does capture only a very weak slowdown upon supercooling and does not show any trace of a critical point, nor any significant improvement for larger $n_{\max }$. The results of multi-component GMCT are discussed more in detail in the next figure; here, we only show that multicomponent GMCT reproduces the binary simulations more realistically than single-component GMCT

the system and therefore dominate the dynamics. Furthermore, as before, we set $k=k_{\text {peak }}$, corresponding to the maximum of $S^{A A}(k)$, thus focusing on the slowest modes in the system $[8,20,25]$.

The results in Fig. 3 show that MCT (red curves, corresponding to GMCT with closure level $n_{\max }=2$ ) overestimates the value of $T_{g}$ obtained from simulations (blue) as expected $[40,41]$. However, if we increase the level of the hierarchy to $n_{\max }=3$ (orange) and then $n_{\max }=4$ (green), the accuracy increases and the critical point of GMCT manifestly converges toward the simulations. This uniform convergence of the multicomponent theory with increasing $n_{\max }$ is also consistent with earlier findings from single-component GMCT $[29,32-34]$.

From the data in Fig. 3, it is particularly noteworthy that at $\rho=1.2$, where the difference between the simulated LJ and WCA dynamics is the largest, higher-order multi-component GMCT becomes increasingly better at distinguishing between the two mixtures $[41,64]$. Here, it is important to recall that for each temperature and density considered, all our GMCT calculations use the same $S^{\alpha \beta}(k)$ as input, regardless of the chosen $n_{\max }$. The fact that increasing $n_{\max }$ leads to better dynamical predictions, and perhaps might even become (near-)exact in the limit of $n_{\max } \rightarrow \infty$ [32], clearly suggests that static 2-point correlations already constitute an important indicator of glassiness - provided that the appropriate dynamical framework is used to translate structure into dynamics. The importance of structural pair correlations has also been verified recently through agnostic machine learning methods [42,65-67], 

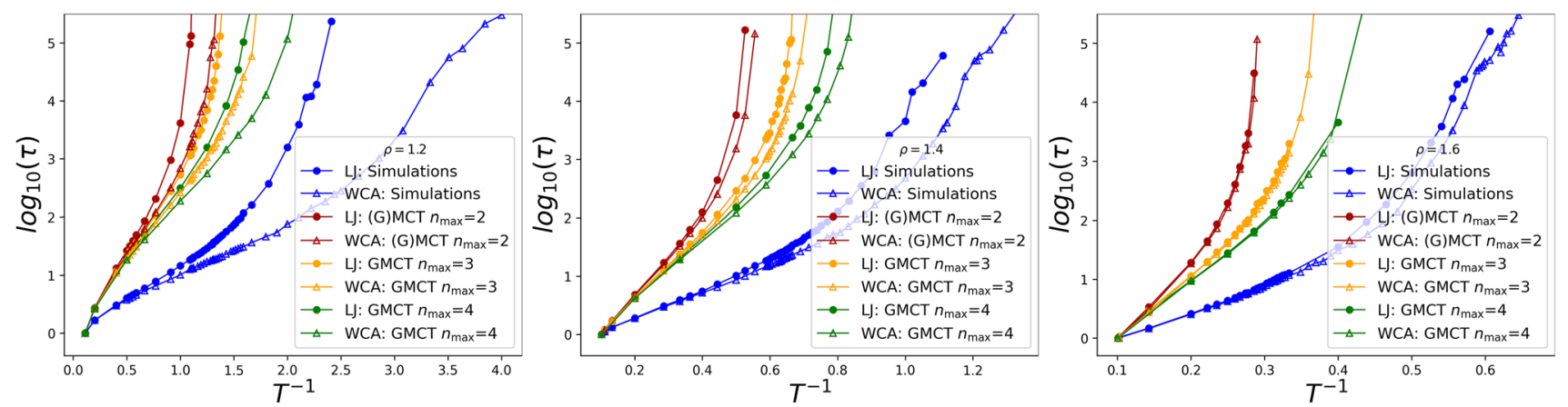

Fig. 3 Relaxation time as a function of inverse temperature from simulations (blue) and multi-component (G)MCT, for binary LJ and WCA mixtures at $\rho=$ $1.2,1.4$, and 1.6. The relaxation time is evaluated from $F^{A A}\left(k_{\text {peak }}, t\right)$, corresponding to the majority species $A$ and

and with the here presented work, we can now place this result on a firmer, first-principles-based theoretical footing.

\subsection{Role of attraction}

The static structure factor $S(k)$ has also been shown to contain information about higher-order static correlations $[44,68]$. However, if we use the relevant $S^{\alpha \beta}(k)$ as the main input of standard MCT, the theory is not able to efficiently distinguish between LJ and WCA mixtures [40,41] (also see Fig. 3). This implies that at least on the MCT level, the role of attractive particle interactions in supercooled liquids is not adequately captured.

We show here that higher-order (multi-component) GMCT is more sensitive to small differences in $S(k)$ and thus the theory is able to recursively recognize better the role of attraction. To support this claim, we compare the LJ and WCA dynamics at different temperatures $T_{\mathrm{LJ}}$ and $T_{\mathrm{WCA}}$, respectively, where the temperatures are defined such that they yield the same relaxation time $\tau$. In Fig. 4, we report the measured temperature difference $T_{\mathrm{WCA}}^{-1}-T_{\mathrm{LJ}}^{-1}$ as a function of the relaxation time $\tau$. This analysis is based on a power law fitting $\tau \sim\left(T-T_{0}\right)^{-\gamma}+A_{0}$, where the parameters $A_{0}, T_{0}$, and $\gamma$ are fitted to best approximate Fig. 3 for each value of $\rho$. All the numerical values are reported in the Supplementary Information (Table S I). It can be seen in Fig. 4 that the temperature difference extracted from the simulations (blue) becomes progressively larger as $\tau$ increases, indicative of the markedly different supercooled LJ and WCA dynamics. In standard MCT, this difference is not properly captured; in fact, binary MCT (red) predicts that the temperature difference is always small and almost constant. However, when we increase the closure level of binary GMCT to $n_{\max }=3$ (yellow) and $n_{\max }=4$ (green) the difference $T_{\mathrm{WCA}}-T_{\mathrm{LJ}}$ becomes larger and, similarly to the simulations, it grows approaching the glass transition. We therefore conclude that higher-order GMCT can extract more information from $S(k)$ and hence it is able to better recognize the role of attraction in the emergent supercooled dynamics. the wavenumber $k=k_{\text {peak }}$ where $S^{A A}(k)$ has its maximum. The data show that by increasing the GMCT closure level, the predictions of multi-component GMCT become increasingly more accurate

\subsection{Conclusions}

In this paper, we have derived generalized MCT for multi-component systems, thus extending the earlier version of the theory $[27-29,33,34]$ to the case of mixtures with an arbitrary number of species. The theory seeks to predict the microscopic relaxation dynamics of glassy mixtures in a fit-parameter-free manner using the static structure factors as its main input. Its hierarchical structure of nested integro-differential equations can be closed and solved self-consistently at any order $n$. The predictive power of the theory manifestly increases for larger $n$, providing a promising, and systematically improvable framework to ultimately achieve an accurate description of the elusive structure-dynamics link in glass-forming liquids.

We have used the newly derived multi-component GMCT to describe the glassy dynamics of threedimensional Kob-Andersen LJ and WCA binary mixtures - systems with almost indistinguishable microstructures but widely different dynamics. We have demonstrated that the theory is able to capture subtle differences in the static structure factors and amplify these to account for the distinct LJ and WCA dynamics. Since the theory only uses $S^{\alpha \beta}(k)$ as input, all the relevant microstructural information is assumed to be fully encoded in the pair-correlations - a result that is consistent with recent machine learning studies on these systems $[42,67]$. Moreover, owing to the improved predictive power of higher-order GMCT compared to standard MCT, we have argued that our theory is also able to better understand the role of attraction in dense supercooled liquids. We have also shown that highly polydisperse systems require a multicomponent theory to properly describe the structuredynamics link in supercooled liquids; this is because the single-component approximation ignores any speciesdependent structural correlations in $S(k)$, thus washing away many subtle but important features in the microstructure that subsequently compromise the predictive power of GMCT.

Lastly, we have illustrated that the systematic inclusion of more levels in the multi-component GMCT hier- 


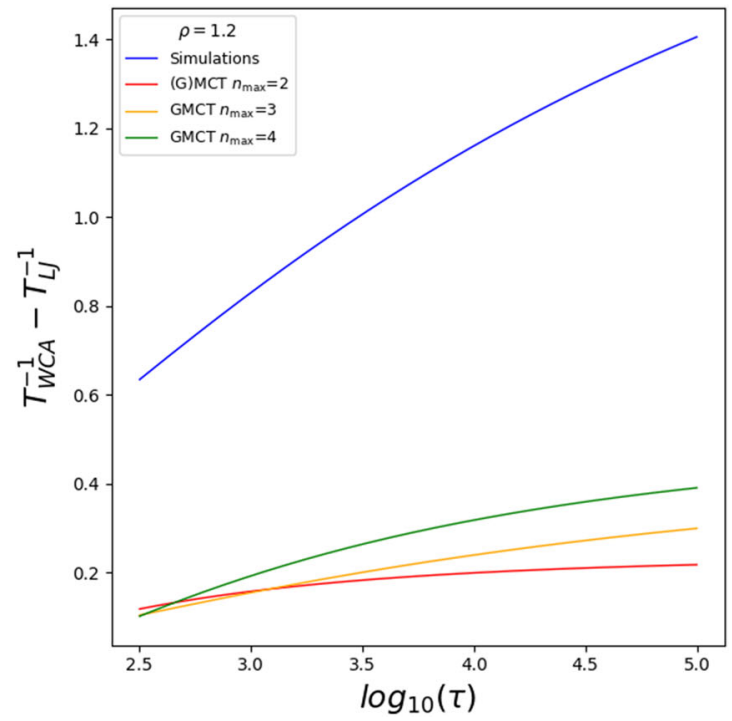

Fig. 4 Effect of the attraction in binary LJ mixtures relative to repulsive WCA, measured in terms of the temperature difference $T_{\mathrm{WCA}}^{-1}-T_{\mathrm{LJ}}^{-1}$ at which both systems yield the same relaxation time $\tau$. The higher the closure level

archy yields quantitatively better predictions for the dynamics, at least based on the first few calculated GMCT levels. This gradual but systematic improvement is also consistent with earlier GMCT studies for single-component systems. In future work, we will aim to push the boundaries of the highest level $n$ we can numerically solve $[33,34]$, in order to check whether the current GMCT framework might approach the exact scenario in the $n \rightarrow \infty$ limit. This effort will also allow us to check if higher-order GMCT can capture the non-trivial glassy phenomenology contained in other successful microscopic theories such as stochastic beta relaxation theory $[21,22]$. To conclude, we hope that our multi-component GMCT could be a useful tool to evaluate how static correlations influence the dynamics of supercooled liquids and to make reliable predictions about the dynamics of such liquids from static information only, thereby contributing to the final understanding of the glass transition.

Supplementary information The online version contains supplementary material available at https://doi.org/ 10.1140/epje/s10189-021-00095-w.

Acknowledgements We thank the Dutch Research Council (NWO) for financial support through a START-UP Grant (C.L., V.E.D., and L.M.C.J.) and Vidi Grant (L.M.C.J.).

\section{Author contribution statement}

SC performed the simulations. SC, CL, and VED developed the theory. SC, CL, and VED analyzed the data.

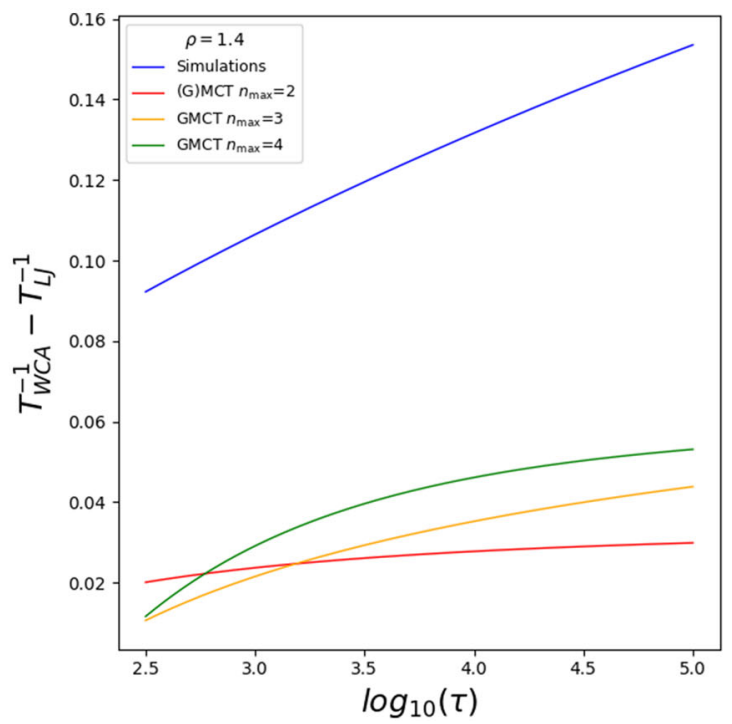

of binary GMCT, the larger this temperature difference becomes. Hence, we can conclude that higher-order GMCT better recognizes the role of attraction via the corresponding changes in the static structure factor

SC, CL, VED, and LMCJ wrote the paper. LMCJ provided resources and supervision.

Open Access This article is licensed under a Creative Commons Attribution 4.0 International License, which permits use, sharing, adaptation, distribution and reproduction in any medium or format, as long as you give appropriate credit to the original author(s) and the source, provide a link to the Creative Commons licence, and indicate if changes were made. The images or other third party material in this article are included in the article's Creative Commons licence, unless indicated otherwise in a credit line to the material. If material is not included in the article's Creative Commons licence and your intended use is not permitted by statutory regulation or exceeds the permitted use, you will need to obtain permission directly from the copyright holder. To view a copy of this licence, visit http://creativecomm ons.org/licenses/by/4.0/.

\section{References}

1. P.W. Anderson, Science 267, 1615 (1995)

2. V. Lubchenko, P.G. Wolynes, Annu. Rev. Phys. Chem. 58, 235 (2007)

3. L. Berthier, G. Biroli, Rev. Mod. Phys. 83, 587 (2011)

4. J.S. Langer, Rep. Prog. Phys. 77, 42501 (2014)

5. G. Biroli, J.P. Garrahan, J. Chem. Phys. 138, 12A301 (2013)

6. P.G. Debenedetti, F.H. Stillinger, Nature 410, 259 (2001)

7. M.D. Ediger, C.A. Angell, S.S.R. Nagel, J. Phys. Chem. 100, $13200(1996)$

8. L.M.C. Janssen, Front. Phys. 6, 97 (2018)

9. C.A. Angell, Science 267, 1924 (1995) 
10. K. Binder, W. Kob, Glassy Materials and Disordered Solids: An Introduction to Their Statistical Mechanics (World Scientific, Singapore, 2011)

11. G. Tarjus, in Dynamical Heterogeneities in Glasses, Colloids, and Granular Media, edited by L. Berthier, G. Biroli, J.- P.Bouchaud, L. Cipelletti and W. van Saarloos (Oxford University Press, 2011) Chap. 2, pp. 39-67

12. A. Cavagna, Phys. Rep. 476, 51 (2009)

13. C.P. Royall, S.R. Williams, Phys. Rep. 560, 1 (2015)

14. G. Biroli, J.-P. Bouchaud, In Structural Glasses and Supercooled Liquids (Wiley, Hoboken, 2012), pp. 31-113

15. X. Xia, P.G. Wolynes, Proc. Natl. Acad. Sci. 97, 2990 (2000)

16. Z.E. Dell, K.S. Schweizer, Phys. Rev. Lett. 115, 205702 (2015)

17. G. Tarjus, S.A. Kivelson, Z. Nussinov, P. Viot, J. Phys. Condens. Matter 17, R1143 (2005)

18. F. Sausset, G. Tarjus, P. Viot, Phys. Rev. Lett. 101, $155701(2008)$

19. W. Götze, L. Sjogren, Rep. Prog. Phys. 55, 241 (1992)

20. D.R. Reichman, P. Charbonneau, J. Stat. Mech. Theory Exp. 2005, P05013 (2005)

21. T. Rizzo, T. Voigtmann, Phys. Rev. Lett. 124, 195501 (2020)

22. T. Rizzo, T. Voigtmann, EPL (Europhys. Lett.) 111, $56008(2015)$

23. W. Götze, Complex Dynamics of Glass-Forming Liquids: A Mode-Coupling Theory (OUP Oxford, 2008)

24. W. Götze, J. Phys. Condens. Matter 11, A1 (1999)

25. W. Kob, in Slow Relaxations and Nonequilibrium Dynamics in Condensed Matter. Les Houches-Ecole d'Ete de Physique Theorique, edited by J. Barrat, M. Feigelman, J. Kurchan and J. Dalibard (Springer Berlin Heidelberg, 2002)

26. G. Biroli, J.P. Bouchaud, K. Miyazaki, D.R. Reichman, Phys. Rev. Lett. 97, 3 (2006)

27. G. Szamel, Phys. Rev. Lett. 90, 228301 (2003)

28. J. Wu, J. Cao, Phys. Rev. Lett. 95, 078301 (2005)

29. L.M.C. Janssen, D.R. Reichman, Phys. Rev. Lett. 115, $205701(2015)$

30. P. Mayer, K. Miyazaki, D.R. Reichman, Phys. Rev. Lett. 97, 095702 (2006)

31. L.M.C. Janssen, P. Mayer, D.R. Reichman, Phys. Rev. E 90, 52306 (2014)

32. L.M.C. Janssen, P. Mayer, D.R. Reichman, J. Stat. Mech. Theory Exp. 2016, 054049 (2016)

33. C. Luo, L.M.C. Janssen, J. Chem. Phys. 153, 214507 (2020)

34. C. Luo, L.M.C. Janssen, J. Chem. Phys. 153, 214506 (2020)

35. L. Klochko, J. Baschnagel, J.P. Wittmer, O. Benzerara, C. Ruscher, A.N. Semenov, Phys. Rev. E 102, 42611 (2020)

36. T. Voigtmann, Europhys. Lett. 96, 36006 (2011)

37. W. Kob, H.C. Andersen, Phys. Rev. Lett. 73, 1376 (1994)

38. A. Ninarello, L. Berthier, D. Coslovich, Phys. Rev. X 7, 021039 (2017)

39. J.D. Weeks, D. Chandler, H.C. Andersen, J. Chem. Phys. 54, 5237 (1971)
40. L. Berthier, G. Tarjus, Phys. Rev. E 82, 031502 (2010)

41. L. Berthier, G. Tarjus, J. Chem. Phys. 134, 214503 (2011)

42. F.P. Landes, G. Biroli, O. Dauchot, A.J. Liu, D.R. Reichman, Phys. Rev. E 101, 10602 (2020)

43. A. Banerjee, S. Sengupta, S. Sastry, S.M. Bhattacharyya, Phys. Rev. Lett. 113, 225701 (2014)

44. D. Coslovich, J. Chem. Phys. 138, 12A539 (2013)

45. G. Nägele, J. Bergenholtz, J.K.G. Dhont, J. Chem. Phys. 110, 7037 (1999)

46. W. Götze, T. Voigtmann, Phys. Rev. E 67, 021502 (2003)

47. T. Voigtmann, Mode Coupling Theory of the Glass Transition in Binary Mixtures (Ph.D. Thesis, Technische Universitat Munchen, 2003)

48. F. Weysser, A.M. Puertas, M. Fuchs, T. Voigtmann, Phys. Rev. E 82, 011504 (2010)

49. C. Ruscher, S. Ciarella, C. Luo, L.M.C. Janssen, J. Farago, J. Baschnagel, J. Phys.: Condens. Matter 33, 034001 (2021)

50. R. Zwanzig, J. Chem. Phys. 33, 1338 (1960)

51. H. Mori, Progress Theoret. Phys. 33, 423 (1965)

52. R.A. Biezemans, S. Ciarella, O. Çaylak, B. Baumeier, L.M.C. Janssen, J. Stat. Mech. Theory Exp. 2020, $103301(2020)$

53. J.-P. Hansen, I. McDonald, Theory of Simple Liquids (Academic Press, New York, 2006)

54. H.W. Jackson, E. Feenberg, Rev. Mod. Phys. 34, 686 (1962)

55. F. Sciortino, W. Kob, Phys. Rev. Lett. 86, 648 (2001)

56. S. Ciarella, "Relaxation pathways for soft materials" (2021)

57. T. Franosch, M. Fuchs, W. Götze, M.R. Mayr, A.P. Singh, Phys. Rev. E 55, 7153 (1997)

58. M. Fuchs, W. Götze, I. Hofacker, A. Latz, J. Phys. Condens. Matter 3, 5047 (1991)

59. J.A. Anderson, C.D. Lorenz, A. Travesset, J. Comput. Phys. 227, 5342 (2008)

60. S. Ciarella, R.A. Biezemans, L.M.C. Janssen, Proc. Natl. Acad. Sci. 116, 25013 (2019)

61. S. Frey, F. Weysser, H. Meyer, J. Farago, M. Fuchs, J. Baschnagel, Eur. Phys. J. E 38, 11 (2015)

62. S.-H. Chong, M. Aichele, H. Meyer, M. Fuchs, J. Baschnagel, Phys. Rev. E 76, 51806 (2007)

63. J. Baschnagel, F. Varnik, J. Phys. Condens. Matter 17, R851 (2005)

64. L. Berthier, G. Tarjus, Phys. Rev. Lett. 103, 170601 (2009)

65. S.S. Schoenholz, E.D. Cubuk, D.M. Sussman, E. Kaxiras, A.J. Liu, Nat. Phys. 12, 469 (2016)

66. E.D. Cubuk, S.S. Schoenholz, J.M. Rieser, B.D. Malone, J. Rottler, D.J. Durian, E. Kaxiras, A.J. Liu, Phys. Rev. Lett. 114, 108001 (2015)

67. J. Paret, R.L. Jack, D. Coslovich, J. Chem. Phys. 152, $144502(2020)$

68. Z. Zhang, W. Kob, Proc. Natl. Acad. Sci. 117, 14032 (2020) 